Canadian Journal of Fisheries and Aquatic Sciences

Canadian Science Publishing

Journal canadien des sciences halieutiques et aquatiques

\title{
Statistical arrival models to estimate missed passage counts at fish weirs
}

\begin{tabular}{|r|l|}
\hline Journal: & Canadian Journal of Fisheries and Aquatic Sciences \\
\hline Manuscript ID & cjfas-2015-0318.R3 \\
\hline Manuscript Type: & Article \\
\hline Date Submitted by the Author: & 04-Dec-2015 \\
\hline Complete List of Authors: & $\begin{array}{l}\text { Sethi, Suresh; US Fish and Wildlife Service, } \\
\text { Bradley, Catherine; US Fish and Wildlife Service, Fisheries and Ecological } \\
\text { Services }\end{array}$ \\
\hline Keyword: & $\begin{array}{l}\text { fisheries management, interpolation, phenology, SALMON < Organisms, } \\
\text { weir }\end{array}$ \\
\hline
\end{tabular}


1 Statistical arrival models to estimate missed passage counts at fish weirs

2 Suresh Andrew Sethi*,1, Catherine Bradley ${ }^{2}$

3

4 *Corresponding author: suresh_sethi@fws.gov, telephone: +1 (907) 786-3655, fax: +1 (907)

$5 \quad 786-3978$

$6 \quad{ }^{1}$ U.S. Fish and Wildlife Service, Fisheries and Ecological Services Division, 1011 E Tudor Road,

7 Anchorage, Alaska 99503, USA.

$8{ }^{2}$ U.S. Fish and Wildlife Service, Fisheries and Ecological Services Division, $10112^{\text {th }}$ Ave,

9 Fairbanks, AK 99701, USA; catherine_bradley@fws.gov

10

11 


\section{Abstract}

13 Missed counts are commonplace when enumerating fish passing a weir. Typically connect-the-

14 dots linear interpolation is used to impute missed passage; however, this method fails to

15 characterize uncertainty about estimates, and cannot be implemented when the tails of a run are

16 missed. Here, we present a statistical approach to imputing missing passage at weirs which

17 addresses these shortcomings, consisting of a parametric run curve model to describe the

18 smoothed arrival dynamics of a fish population and a process variation model to describe the

19 likelihood of observed data. Statistical arrival models are fit in a Bayesian framework and tested

20 with a suite of missing data simulation trials and against a selection of Pacific Salmon

21 (Oncorhynchus spp.) case studies from the Yukon River drainage, Alaska, U.S.A. When

22 compared against linear interpolation, statistical arrival models produced equivalent or better

23 expected accuracy and a narrower range of bias outcomes. Statistical arrival models also

24 successfully imputed missing passage counts for scenarios where the tails of a run were missed.

25

26 Keywords: fisheries management, interpolation, missing data, phenology, salmon 


\section{Introduction}

Fish which migrate en masse along river systems during part of their life history provide opportunity to census populations by directly counting passage at a weir. Weirs are relied upon

31 heavily in harvested salmonid systems to provide information for run reconstruction and to guide

32 fishery management decisions, with weir sites typically sighted in upper reaches of river

33 networks and providing estimates of adult spawning escapement after passing through a harvest

34 gauntlet down river (e.g. Fleischman et al. 2013). While typically used to assess adult fish

35 migrating into river systems, they can also be used to assess the abundance of out-migrating

36 juvenile fish such as Pacific Salmon smolts (Oncorhynchus spp.; Bradford 1999).

37 In theory, weirs produce complete enumeration of a fish run passing a point in space,

38 providing a gold standard for accurate enumeration data. In practice, field crews contend with

39 myriad challenges in successfully installing and operating a weir-high water can blow a weir

40 out or make it unsafe to operate, woody debris can overwhelm weir structures, river ice can delay

41 the installation of weirs or require their early removal, and bears can vandalize weirs or harass

42 weir operators (Figure 1). Thus, missing data at weirs are a common occurrence leading to

43 partial enumeration of fish populations.

Attempts to impute missing data at weirs typically involve a "connect-the-dots" linear

45 interpolation scheme (e.g. Gewin et al. 2005; Johnson et al. 2007). This approach is attractive in

46 that it is simple to implement; however, linear interpolation requires a passage observation

47 before and after a missing datum and thus is not an option to impute missing data in the tails of a

48 run when weirs are installed late or pulled early. Furthermore, linear interpolation does not

49 produce estimates of uncertainty for imputed missing passage counts, potentially communicating

50 false precision to users of the data. Another, less frequently adopted, approach to imputation 
51 which can be implemented with standard spreadsheet software utilizes an expectation-

52 maximization algorithm to combine run information across years to impute passage at specific

53 dates, including in the tails of the run (e.g. Van Alen 2000). The expectation-maximization

54 algorithm based approach asserts run dynamics from observed years to dates with missed

55 passage. As such, the approach necessitates multiple years of data and requires the restrictive

56 assumption that the proportion of a run that passes on a given date is constant across years.

57 An alternative to linear interpolation or expectation-maximization algorithm based

58 imputation is to take an arrival model based approach and implement a statistical framework

59 with which to make inference about passage counts at a weir. Several related efforts have been

60 developed for estimating abundance of fish in streams associated with periodic ground-based

61 stream surveys (e.g. Hilborn et al. 1999; Su et al. 2001). These applications explicitly model the

62 arrival of fish in streams and the "decay" dynamics as fish exit a stream (i.e., become unavailable

63 for detection) through depredation by predators, decomposition, or emigration. The decay model

64 is necessary to avoid double counting of previously observed fish. Subsequently, a probability

65 model is asserted to describe variability of observations about the underlying arrival and decay

66 models describing fish presence in the stream, providing a likelihood for observed fish counts.

67 Total stream abundance enters the model framework as a scalar on the arrival and decay

68 processes and is estimated using the likelihood for the observed data. Hilborn et al. (1999)

69 implemented the approach using maximum likelihood estimation, and Su et al. (2001) extended

70 the approach into a hierarchical Bayesian framework allowing for analysis of multiple years of

71 data in manner which captures across-year correlation in stream arrival and decay processes.

72 While these approaches deal with stream surveys on Pacific Salmon with an additional

73 complexity of needing to correct observations for the fact that fish persist in stream for some 
74 variable amount of time, much of the underlying approach is applicable to imputation of missing 75 data at weirs.

Conceptually, weir-based fish counts are simpler than ground-based stream surveys

77 because weir passage is a unidirectional, one-time event. As such, an arrival model can be

78 specified to describe the passage of a run of fish at the weir; however, a decay model which

79 corrects for potential double counting of previously observed fish is unnecessary. Once fit, the

80 arrival model and assessment of variability of realized passage counts about the smooth

81 underlying arrival model can be used to estimate passage counts for missing dates. For

82 example, the arrival model of fish at a weir could be specified as following a cumulative Normal

83 distribution-shaped curve, and the deviations about the smooth arrival model specifying actual

84 weir passages could be modeled as Negative Binomial random variables. Subsequently, the

85 Negative Binomial likelihood model can be used to inform best fitting parameters describing the

86 arrival curve for a dataset of daily passage counts, and ultimately make predictions about missing

87 passage dates. Because the approach is based upon a statistical framework, uncertainty about

88 missing passage estimates can be assessed, for example by providing confidence (or credibility)

89 intervals for missed passage estimates.

90 In this article, we introduce a parametric statistical approach based upon fitted run arrival

91 models in order to impute missing data and estimate total passage at weirs. Models are fit in a

92 Bayesian framework, providing a straightforward means to summarize uncertainty about total

93 run size estimates, arrival model characteristics (e.g. peak run date), and estimates of predicted

94 passage counts on missing-observation dates. Subsequently, information-theoretic model

95 selection is used to assess strength of evidence for different proposed arrival models and

96 observation processes. Estimator performance is tested by simulating weir data modeled after 
97 Pacific Salmon run dynamics under a suite of missing data scenarios including missing tails,

98 missing peak run days, and data collection efforts of only 5 days a week (the "take weekends

99 off" scenario). Performance of parametric run models is compared against a simple linear

100 interpolation, “connect-the-dots" scheme. Finally, the parametric run curve approach is

101 illustrated on observed weir data for Pacific Salmon runs in the Yukon River, Alaska, drainage.

102 We find that statistical arrival models can be successfully applied to particularly ill-behaved

103 datasets (e.g. missing observations from the tails of the run curve) where linear interpolation can

104 fail dramatically. Furthermore, parametric models provide statistical estimates of arrival

105 dynamics parameters informative for assessing fish phenology.

107 Methods

108 The statistical framework implemented to estimate missing data at weirs requires

109 specification of two key processes: $i$ ) an underlying run curve model that governs fish arrival

110 dynamics at the weir, and ii) an error model that governs the noise about the smooth arrival

111 model exhibited by a realized fish run and which serves as the likelihood model for the observed

112 weir counts. Subsequently during model fitting, the parameters specifying the shape of the

113 arrival model, a total run size scalar for the arrival model (see below), and the parameters

114 specifying the error process model are estimated. Statistical arrival models are implemented in a

115 Bayesian framework, providing a means to directly estimate missing data while accounting for

116 uncertainty by treating missing daily passage counts as derived parameters for which posterior

117 summaries are generated. The key parameter of interest, total run size, is defined as a derived

118 parameter equal to the sum of all estimated missing daily passage counts plus the known (i.e.

119 observed) passage counts. 


\section{$121 \quad$ Statistical models}

122 Passage dynamics at the weir are specified by a run arrival curve model that describes the

123 cumulative proportion, $p_{t}$, of the run that has passed the weir at time step, $t$. In what follows, we

124 define a time step as a 24 hour day; any choice of time step may be implemented, however,

125 passage counts need match the specified time step and daily counts are the most commonly

126 recorded weir data. We implemented three candidate arrival models: a run curve described by

127 the function for a Normal cumulative distribution,

$129 p_{t}=F_{N}(t \mid \mu, \sigma)=\int_{-\infty}^{t} \frac{1}{\sigma \sqrt{2 \pi}} e^{-\frac{(x-\mu)^{2}}{2 \sigma^{2}}} d x$

eq. 1,

130

131 a left or right skewed run curve described by the function for a skew-Normal cumulative

132 distribution (Azzalini 1985),

$134 p_{t}=F_{S N}(t \mid \xi, \omega, \alpha)=\frac{2}{\omega} \phi((t-\xi) / \omega) \Phi(\alpha(t-\xi) / \omega)$

eq. 2,

136 and a fat-tailed symmetric run curve described by the function for a Student's t cumulative

137 distribution,

138

139

$p_{t}=F_{S t}(t \mid \delta, \gamma, v)=\int_{-\infty}^{t} \frac{\Gamma\left(\frac{v+1}{2}\right)}{\Gamma\left(\frac{v}{2}\right) \gamma \sqrt{\pi v}}\left[1+\frac{1}{v}\left(\frac{x-\delta}{\gamma}\right)^{2}\right]^{-(v+1) / 2} d x$

eq. 3, 
141 where $F_{N}, F_{S N}$, and $F_{S t}$ are notation for the Normal, skew-Normal, and Student's t cumulative

142 distribution functions, respectively, $\mu$ is the location parameter and $\sigma$ is the scale parameter for

143 the Normal distribution arrival model, $\xi$ is the location parameter, $\omega$ is the scale parameter $\alpha$,

144 is the shape parameter (describing skewness) for the skew-Normal distribution, $\phi$ is the standard

145 normal density function, and $\Phi$ is the standard Normal cumulative distribution function, $\delta$ is the

146 location parameter, $\gamma$ is the scale parameter, and $v$ the shape (degrees of freedom) for the

147 Student's t distribution. Subsequently, daily passage counts under the arrival model, $c_{t}$, are

148 calculated by taking the proportion of the run that arrives over a preceding time step multiplied

149 by a total run size scalar, $S$ :

150

$151 \quad c_{t}=S \times\left(p_{t}-p_{t-1}\right)$

eq.4.

152

153 To avoid confusion, it is worth emphasizing that the cumulative distribution functions used to

154 specify arrival dynamics do not represent probability models for weir passage counts; they are

155 merely convenient mathematical functions to describe the shape of the arrival curve of fish at a

156 weir.

157 Next, a likelihood for the observed passage counts is specified by asserting a probability

158 model for the process variation about passage counts predicted under the arrival model. We

159 considered both a Normal and Negative Binomial probability model for an observed passage

160 count, $c_{t}^{o}$ :

161

$162 c_{t}^{o} \sim \operatorname{Normal}\left(c_{t}, \tau\right)$

eq. 5 , 
164 or

165

$166 c_{t}^{o} \sim$ NegativeBinomial $\left(\lambda=\theta /\left(\theta+c_{t}\right), \theta\right)$

eq.6,

167

168 where the specified parameterization of the success probability parameter, $\lambda$, in the Negative

169 Binomial model produces an expectation of $c_{t}^{o}$ (i.e. observed passage) equal to $c_{t}$ (i.e. passage

170 under the smooth arrival curve) as a function of the dispersion parameter $\theta$ (e.g. Ntzoufras

171 2009). The Normal process variation model implies that variability in realized passages about

172 the smooth underlying arrival curve is constant across the run, whereas the Negative Binomial

173 model assumes that passage count variability scales positively with the magnitude of passage.

174 We considered white noise process variation models (eqs. 5-6) and those with lag-1

175 autoregressive $(\mathrm{AR}(1))$ and moving average $(\mathrm{MA}(1))$ serially correlated process variation (e.g.

176 Greene 2008). Normal and Negative Binomial probability models for observed passages with

$177 \mathrm{AR}(1)$ process variation are represented as, respectively:

178

$179 \quad c_{t}^{o} \sim \operatorname{Normal}\left(c_{t}+\rho_{A R(1)} c_{t-1}^{*}, \tau\right)$

eq. 7 ,

180

181 or

182

$183 c_{t}^{o} \sim$ NegativeBinomial $\left(\lambda_{A R(1)}=\theta /\left(\theta+c_{t}+\rho_{A R(1)} c_{t-1}^{*}\right), \theta\right) \quad$ eq.8, 
185 where $\rho_{A R(1)}$ is the AR(1) serial correlation coefficient, $c_{t-1}^{*}=c_{t-1}^{o}$ if passage was observed on

186 date $t-1$, else $c_{t-1}^{*}=c_{t-1}$, i.e. the predicted passage count at time $t-1$. Similarly, Normal

187 and Negative Binomial models for observed passages with MA(1) process variation are,

188 respectively:

189

190

$c_{t}^{o} \sim \operatorname{Normal}\left(c_{t}+\rho_{M A(1)}\left(c_{t-1}^{*}-c_{t-1}\right), \tau\right)$

eq. 9 ,

191

192 or

193

194

$c_{t}^{o} \sim$ NegativeBinomial $\left(\lambda_{M A(1)}=\theta /\left(\theta+c_{t}+\rho_{M A(1)}\left(c_{t-1}^{*}-c_{t-1}\right)\right), \theta\right) \quad$ eq.10,

195

196 where $\rho_{M A(1)}$ is the $\mathrm{MA}(1)$ serial correlation coefficient and with $c_{t-1}^{*}$ defined as above. A key

197 difference between the forms of serial correlation, is that imputed missing passage counts in an

198 AR(1) model contain lagged influence from all earlier observed passage counts in the data set,

199 whereas imputed missing passage counts in the MA(1) model contain influence only from

200 observed passage counts one time step earlier if such an observed passage count is available

201 (otherwise the prediction is simply that as predicted by the fitted smooth underlying arrival

202 curve). Depending on the specified arrival model and process error model combination,

203 likelihoods of observed counts, $L\left(\boldsymbol{c}^{o} \mid \boldsymbol{\beta}\right)$ are conditional upon four to six parameters, $\boldsymbol{\beta}$

204 (Supplement 1, Supplementary Materials).

205

206 Model fitting 
208 algorithms as implemented in the WinBUGS software platform (Lunn et al. 2000) and run from

209 the R statistical programming environment (RDCT 2014) using the package R2WinBUGS

210 (Sturtz et al. 2005). Example code is provided in Supplement 2 of the Supplementary Materials.

211 Diffuse priors were specified for fitted parameters (Table 1). WinBUGS treats missing daily

212 passage counts as parameters to be estimated and thus returns a posterior distribution for the

213 passage estimate for each missing date. The sum of all observed passages and of all estimated

214 passages for missing dates was treated as a derived parameter, returning a posterior distribution

215 for the estimated total run size. Because observed passage counts are known quantities, this

216 estimate of total run only incorporates uncertainty about estimated missing passage counts.

217 WinBUGS does not have native skew-Normal or Student's t cumulative distribution functions.

218 Thus, we implemented a numerical integration routine within WinBUGS models to approximate

219 these cumulative distribution functions following equations 2-3 (Supplement 2, Supplementary

220 Materials).

To improve model fitting, we included additional prior information when fitting

222 statistical arrival models by including two dates approximately one month before the start of the

223 run and two dates approximately one month after the end of the run with zero passage counts.

224 Preliminary analyses indicated that inclusion of known zero passage dates improved estimation

225 of arrival models, particularly when observed data was missing for the tails of the run as may

226 occur when a weir begins operation after the commencement of a run or is pulled before the

227 close of a run. The timing of these dates were selected to be far enough before and after the run

228 such that there is high confidence they represent true zero passage dates; these represent a form

229 of prior knowledge because a priori, the timing of the fish run to be modeled isn't known, 
230 however, analysts typically have enough information about the timing of a run to propose dates

231 early enough and late enough to be true zero passage days. We conducted simulations to test the

232 sensitivity of estimation results to the number and placement of known zero passage dates and

233 found total run size estimates to be robust (Supplement 3, Supplementary Materials).

235 Performance testing

236 We compared the performance of the statistical arrival models against a suite of trials in

237 which we simulated fish runs modeled after Pacific Salmon data from the Yukon River (Table

238 2). The basic approach to performance testing involved three phases: $i$ ) assert "true" fish run

239 arrival dynamics and simulate realized fish runs with process variation, $i$ ) implement observed

240 fish runs which include missing passage counts (e.g. the initial 15\% of the run went unobserved),

241 and iii) estimate missing passage counts and summarize performance.

242 Simulation trials included several plausible scenarios weir operations might experience in

243 the field (Table 2), including scenarios where the peak of the run was missed (+/- 2 days about

244 peak run day), scenarios where tails of the run were missed (initial 15\% of run missed), and a

245 scenario of sampling five days a week with weekends off. White noise, AR(1), and MA(1)

246 serially correlated process variation was simulated. In order to constrain the number of

247 simulation scenarios, a selection of Normal and skew-Normal arrival dynamics were simulated;

248 models with Student's t distribution arrival dynamics were not used in performance testing but

249 were fit to case study data (see below).

250 Simulated daily and total passage counts were calculated by asserting a smooth arrival

251 process and then adding process variation noise, following the approach outlined above: latent

252 daily passage counts are calculated as the proportion of an asserted arrival curve that passes on a 
253 given date multiplied by a total run size scalar, and subsequently, realized daily passages are

254 drawn from probability distributions with expectations equivalent to the latent passage count.

255 We fixed the total run size scalar at 10000 fish for each simulated data set, but because realized

256 true daily passages included process noise, realized total run size typically differed from 10000.

257 Accordingly, subsequent performance of estimators for run size with missing passage counts was

258 assessed relative to the realized true run size for a given simulated data set.

259 Salmon data from Yukon River weirs suggests that daily passage variability scales

260 positively with passage magnitude (e.g. Figure 2), therefore simulated data scenarios

261 incorporated Negative Binomial process variation about a given arrival model. The amount of

262 process variation specified for simulated fish runs was chosen to reflect variation observed in

263 actual Yukon River Pacific Salmon data, and was fixed at $\theta=7.0$. Model fits to Yukon River

264 Pacific Salmon datasets which supported lag-1 serially correlated process variation indicated a

265 wide range of positive serial correlation coefficients centered about 0.5 . Lag-1 serially

266 correlated process variation was included in a selection of data simulations, setting the serial

267 correlation coefficient, $\rho=0.5$, reflecting a moderate amount of positive autocorrelation.

268 For each simulated fish run, the parameters describing the arrival model functions were

269 randomly drawn from probability distributions, such that each simulated fish run had a unique

270 shape and timing. Location parameters for both Normal and skew-Normal arrival models were

271 drawn from a Normal distribution with a mean of day 185 (i.e. Julian date corresponding to July

2724 in non leap years) and standard deviation of 2.5 days. Scale parameters for both Normal and

273 skew-Normal arrival models were drawn from a log-Normal distribution with a mean of $\ln (7.5)$

274 and standard deviation of $\ln (1.1)$. Skewness aside, these distributions for fish run arrival timing

275 (location parameters) and fish run compression (scale parameters) result in runs of approximately 
27640 days in length with a peak around July 4. Finally, for simulation scenarios with skew-Normal

277 arrival curves, the shape parameter describing skewness was fixed at a magnitude of 2.0 with the

278 direction of skew randomly chosen for each simulated run (i.e. $\alpha$ is either -2.0 or 2.0), providing

279 a moderate amount of skewness to simulated fish runs (Figure2c). Note, because the direction of

280 skew was randomly chosen, simulation scenarios representing late-entry of weirs where the

281 initial $15 \%$ of the run was missed are implemented for both left- and right-skew runs.

282 For each simulated data set with white noise process variation, we fit four statistical

283 models with serially independent process variation — a Normal arrival curve with Normal

284 deviates or with Negative Binomial deviates, and a skew-Normal arrival curve with Normal

285 deviates or with Negative Binomial deviates - to the simulated observed data and estimated

286 missing data following Bayesian implementation detailed above (Table 2). For simulations to

287 examine the implication of lag-1 serially correlated process variation, we fit six statistical

288 models - Normal arrival curve with serially independent, AR(1), and MA(1), Negative Binomial

289 deviates, and skew-Normal arrival curve with serially independent, AR(1), and MA(1), Negative

290 Binomial deviates (Table 2). Preliminary model analyses indicated Markov chains converged

291 quickly. Subsequently, two parallel chains each with a 5000 iteration burn in period, a thin rate

292 of 10 iterations, and 1000 posterior parameter draws stored were implemented for each model fit.

293 Model convergence was assessed using the Gelman-Brooks-Rubin $\hat{R}$ statistic (Brooks and

294 Gelman 1998) for the key parameter of interest, derived total run size estimate (i.e. observed

295 passages plus the sum of imputed missing passage counts).

In addition to statistical arrival models, we implemented a "connect the dots" linear

297 interpolation estimator commonly used to impute missing data at weirs. The "connect the dots"

298 approach is attractive because of its simplicity to implement, however the approach does not 
299 characterize the uncertainty about estimates of missing passage counts. With this estimator,

300 missing passage day are estimated using the slope, $m$, of a straight line between the observed

301 passage count days nearest to a missing day or sequence of missing days:

302

303

$\widehat{m}_{t_{a}, t_{b}}=\left(c_{t_{b}}^{o}-c_{t_{a}}^{o}\right) /\left|t_{b}-t_{a}\right|$

eq. 10,

304

$\hat{c}_{t}=c_{t_{a}}^{o}+\left(t-t_{a}\right) \hat{m}_{t_{a}, t_{b}}$

eq. 11 ,

305

306 where “^” indicates an estimated quantity, $t_{a}$ is the nearest date with an observed passage count

307 preceding a given missing-observation passage day, and $t_{b}$ the nearest date with an observed

308 passage count following a given missing-observation passage day. The "connect the dots"

309 estimator requires dates with observed passage counts both before and after a given missing date,

310 and thus cannot directly interpolate missing tails of the run without additional assumptions. We

311 were unable to identify a standard approach to linearly impute missing data in the tails of runs

312 from published literature. Therefore, we implemented an approach whereby the slope parameter

313 for linear imputation is estimated by drawing a straight line from the maximum observed passage

314 day (as an empirical estimate of the peak of the run) to the first observed passage day in cases

315 when the initial tail of the run is missing, or to the last observed passage day in cases where the

316 terminal tail of the fish run is missing. Subsequently, cumulative passage in the missing

317 tail, $\hat{c}_{\text {tail }}$, is estimated by taking the area of the triangle formed by extrapolating the estimated

318 slope to a point of zero passage before (after) the first (last) observed passage date when the

319 initial (terminal) tail of the run is missing: 
$321 \hat{m}_{\text {tail }}=\left(c_{\max }^{o}-c_{t^{*}}^{o}\right) /\left|t_{\max }-t^{*}\right| \quad$ eq. 12,

$322 \hat{c}_{\text {tail }}=\left(c_{t^{*}}^{o}\right)^{2} / 2 \hat{m}_{\text {tail }}$

324 where $c_{\max }^{o}$ is the maximum observed passage count which occurs on date $t_{\max }$, and $c_{t^{*}}^{o}$ is the

325 first (last) observed passage count on date $t^{*}$ when cumulative passage in the initial (terminal)

326 tail of the run is to be estimated.

327 A total of 150 data sets were generated for each simulation scenario (Table 2). Simulated

328 datasets for which one or more of the fitted statistical models resulted in $\widehat{R}>1.2$ for the total run

329 size estimate, or for which the linear interpolation estimator failed (see below), were dropped

330 from consideration. Estimator performance was measured as average percent bias, interquartile

331 range in percent bias, and root mean squared error relative to the true realized run size. In

332 addition, for statistical estimators implemented in a Bayesian approach, we report the median

$33395 \%$ highest posterior density intervals (HPDI) for the derived estimate of the total run. Finally,

334 we used DIC-based model selection (Spiegelhalter et al. 2002; Celeux et al. 2006) to assess

335 whether the "correct" statistical model which matched the simulated arrival and process noise

336 model was identified as the best fitting model. We calculated the model complexity penalty in

337 DIC using one half the variance of the posterior deviance, following an approach outlined in

338 Gelman et al. (2004; Section 6.7).

340 Case studies

341 Preliminary model runs indicated that the degrees of freedom parameter for Student's t

342 arrival models were poorly identified, insofar that posterior distributions looked very similar to 
343 prior distributions and were sensitive to specification of prior distributions; subsequently,

344 Student's t arrival dynamics were fit with the degrees of freedom parameter fixed to 2.0 (heavy

345 leptokurtosis). A suite of 18 statistical estimators for all combinations of 3 arrival models

346 (Normal, skew-Normal, and Student's t with $v=2.0$ ), 2 process variation models (Normal and

347 Negative Binomial), and 3 forms of serial correlation (white noise, AR(1), and MA(1)), and the

348 linear imputation approach to estimating missing data at weirs were tested against a selection of

349 Pacific Salmon data sets with missing passage dates from resistance board weirs operated by the

350 U.S. Fish and Wildlife Service on the Gisasa and East Fork Andreafsky rivers in the Yukon

351 River drainage, Alaska, USA, during 1998 and 2000 (Tables 2-3; data publicly available from

352 the U.S. Fish and Wildlife Service, email: ak_fisheries@fws.gov). Yukon River salmon were

353 selected as a study system because weir operations in this high latitude, large river system often

354 experience challenges with blowouts from high water and difficulty in installing (maintaining)

355 weirs early (late) in the season due to ice. Furthermore, salmon in these systems follow the

356 typical unimodal arrival curve for migrating Pacific Salmon (Figure 2). Case study datasets were

357 chosen to represent a selection of missing data scenarios, run sizes, and Pacific Salmon species

358 (Chinook: O. tshawytscha; Chum: O. keta; Coho: O. kisutch; Pink: O. gorbuscha) datasets were

359 selected prior to completion of simulation analyses. A priori placement of zero passage days

360 (Table 3) were made based on the shape of the arrival curve or, in the instance where one or both

361 tails were unobserved, by referencing data collected in previous years from a given system.

362 Sensitivity analyses indicated total run size estimates for case studies were robust to zero passage

363 day placements. Two parallel chains each with a 7500 iteration burn in period, a thin rate of 10

364 iterations, and 1500 posterior parameter draws stored were implemented for model fits. The 
365 same uninformative priors and convergence diagnostics used to fit statistical arrival models for 366 data simulations (above) were also used for case study data (Table 1).

\section{Results}

369 Simulations: white noise process variation

370 Total run size estimation performance varied across models and simulation scenarios

371 with white noise process variation; however, in most cases missing passage estimates could be

372 imputed with accuracy and reasonable precision. The 'Weekends Off' scenario resulted in

373 accurate and precise missing passage imputation estimates across all estimators with either

374 Normal or skew-Normal arrival dynamics (Table 4; "Weekends off (SN)" and "Weekends off

375 (SN)" scenario). In these scenarios, maximum average bias was only $-1.1 \%$ of true run size for

376 the $S N-N$ estimator fit to $S N-N B$ arrival dynamics. Statistical estimators indicated precise total

377 run size estimates with 95\% HPDI half widths on the order of $\pm 14-19 \%$ of total missed passage

$378( \pm 4-5 \%$ of total run size). DIC support was strongest for the statistical arrival model - process

379 variation combination that matched that from the data generating process. In the case of the

380 Normal arrival dynamics “Weekends off” scenario, DIC support was indicated for a $S N-N B$

381 model in $30 \%$ of the trials; however, the average difference in DIC between the $N-N B$ model and

382 the $S N-N B$ model in these cases was 0.04 DIC units, indicating that the two models were

383 comparably supported by the data (data not shown but available from the authors).

384 Imputation of missing passage counts on scenarios with missed peak run days was

385 generally successful for both the Normal and skew-Normal arrival curve data generating

386 processes simulated. Accuracy and precision of total run estimates were comparable for data

387 simulated under the Normal and skew-Normal arrival models. For example, expected bias of the 
388 DIC-preferred model $(N-N B)$ under the Normal arrival curve scenario was $-0.3 \%$ with a HPDI 389 half-width of approximately $\pm 19 \%$ of total missed passage, and the DIC-preferred model (SN$390 N B$ ) under the skew-Normal arrival curve scenario resulted in slightly larger expected bias of $3912.6 \%$ with HPDI half-width of approximately $\pm 24 \%$ of total missed passage (Table 4 ; “ \pm 2 days 392 about the peak missed" scenarios). In both scenarios, DIC-based model selection identified the 393 correct approximating model the majority of time. Linear interpolation imputation estimators 394 had performance within the range observed for statistical arrival curve estimators; however, the 395 former was never the best estimator in terms of expected bias, range in bias, and RMSE.

396 Simulation scenarios for which the initial 15\% of the run was missed-representing, for 397 example, late installation of a weir in the field - presented a greater range in estimator 398 performance and lower precision when compared against scenarios with missing passage dates 399 within the interior of arrival curves. Accuracy was generally higher for missing tail scenarios 400 with Normal arrival dynamics, resulting in expected biases ranging from 0.5 to $11.4 \%$ across 401 estimators, as compared to missing tail scenarios with skew-Normal arrival dynamics, which 402 resulted in expected biases ranging from 6.3 to $17.4 \%$. DIC-based model selection performed 403 well for the Normal arrival curve scenario, correctly identifying the $N-N B$ model as the best fit 404 model in over 80\% of trials (Table 4; "First 15\% missed (Normal)" scenario); however, DIC405 based model selection in the case of skew-Normal arrival dynamics indicated ambiguity, 406 supporting both the $N-N B$ and $S N-N B$ as preferred models (Table 4; "First 15\% missed (skew407 Normal)" scenario). Because of the good performance of the $S N-N B$ model for missing-tail data 408 scenarios for both Normal and skew-Normal arrival dynamics and the potential for a skew409 Normal model to accommodate both skewed and symmetric run shapes (i.e. shape parameter $410 \alpha=0$ ), in cases of ambiguous DIC-based model support we recommend implementing the $S N$ - 
$411 N B$ model when handling real data with missed passage counts for tails of unimodal-shaped runs

412 such as for Pacific Salmon enumeration as examined herein. Finally, linear interpolation

413 estimators produced average percent bias comparable to the best fit statistical models for missing

414 tail scenarios; however, linear interpolation could result in very large positive bias outcomes with 415 maximum percent bias of $176.6 \%$ in the case of Normal arrival dynamics and maximum bias of $416930 \%$ in the case of skew-Normal dynamics (Table 4).

\section{Simulations: serially correlated process variation}

419 Arrival models fit to simulation scenarios for which the initial $15 \%$ of the run was missed 420 and which included serially correlated process variation performed comparably in terms of bias

421 to equivalent simulation scenarios with white noise process variation, enjoying good expected

422 accuracy and a relatively tight range in bias outcomes (Table 5). Highest posterior density

423 interval widths for models fit with serially correlated process variation were greater than

424 equivalent arrival models fit with white noise process variation, indicating that naïve reliance on

425 white noise models when serial correlation is present in a given data set may result in an

426 underestimation of the uncertainty about imputed missed passage (Table 5). While DIC-based

427 model selection produced high success in identifying the correct arrival curve model under data 428 simulated with white noise process variation (e.g. Table 5 scenario "First 15\% missed (N-WN)"),

429 DIC based model selection performed more poorly with serially correlated simulation data,

430 indicating comparable support for white noise and serially correlated process variation models

431 (e.g. Table 5 scenario "First 15\% missed (N-AR(1))"). That said, practical differences in bias

432 performance and HPDI widths were small across the AR(1) versus MA(1) forms of serially

433 correlated process variation. Furthermore, arrival curves fit with serially correlated process 
434 variation to data simulated with white noise process variation (Table 5 scenario "First 15\%

435 missed (N-WN)") indicated comparable bias performance as the "correct" white noise model,

436 and the inflation in subsequent highest posterior interval widths was slight. In such cases when

437 candidate models with serially correlated process variation were fit to data simulated with white

438 noise process variation (i.e. Table 5 scenario "First 15\% missed (N-WN)"), serial correlation

439 coefficients were correctly estimated as close to 0.0 (Table S2). Thus, when faced with

440 ambiguous DIC support for white noise versus serially correlated process variation, we

441 recommend erring on the side of caution and implementing models with serial correlated process

442 variation on the grounds of comparable expected bias performance, and conservative

443 characterization of uncertainty about imputed passage counts.

444 The performance gap between statistical arrival models and linear interpolation widened

445 with inclusion of serially correlated process variation when considering simulation scenarios for

446 which the initial $15 \%$ of the run was missed. Both expected bias and maximum observed bias

447 across simulation trials increased substantially for the linear interpolation estimator when faced

448 with $\mathrm{AR}(1)$ or $\mathrm{MA}(1)$ serially correlated process variation and a missing tail scenario. In some

449 cases, the proposed rule of thumb for linearly interpolating tails of runs produced bias outcomes

450 of over 10,000\% (e.g. Table 5 scenario "First 15\% missed (N-MA(1))").

451

452 Simulations: arrival curve attributes

453 While not a focus of the present simulation trials, statistical arrival models performed

454 well in reconstructing underlying run curve characteristics (see summary of full results in

455 Supplementary Materials, Table S1-S2). Arrival curve location and spread parameters were

456 estimated with high accuracy, with expected bias for peak run date estimated to within \pm 1 day 
457 and spread parameters to within $\pm 5 \%$ of the true estimate for DIC-preferred models. Arrival 458 curve skew, which was constrained to 2.0 or -2.0 during simulations, was accurately estimated

459 with average absolute bias of 0.1 for the $S N-N B$ model applied to the missing peak days

460 scenarios with skew-Normal arrival and white noise Negative Binomial process variation, and 461 average absolute bias of 0.3 for the $S N-N B$ model applied to missing $15 \%$ initial tail scenario

462 with skew-Normal arrival. Similarly, $S N-N B$ models fit to missing data scenarios with Normal 463 arrival dynamics and white noise Negative Binomial process variation produced average

464 skewness estimates close to zero (average absolute value of shape parameter ranged from 0.3 to 4650.6 across missing data scenarios; Table S1). Finally, expected estimates of the degree of serial 466 correlation in the process variation models were accurate for white noise (average $\rho \approx 0.0$ ), as 467 well as AR(1) and MA(1) serially correlated Negative Binomial process variation models 468 (average $\rho \approx 0.5$; Table S2).

470 Case Studies

471 The $S N-N B$ was the DIC-preferred model in all but one case study, indicating substantial

472 left and right skew in 4 of 5 of the case studies (Table 6; Figure 2d-h). In one case, Student's t

473 distribution with two degrees of freedom was DIC preferred (Table 6, "2000 East Fork

474 Andreafsky River Pink Salmon). A mix of both white noise and serially correlated process

475 variation was supported, depending on the case study. Based upon total run size from the DIC-

476 preferred statistical arrival model, imputed passage counts ranged from $15-31 \%$ of the total run

477 (Table 6). Precision of total run estimates varied, and was not strongly related to the amount of

478 missing passage imputed or the shape of the run. For example, the $S N-N B-M A(1)$ statistical

479 arrival estimator indicated $26 \%$ of the Chum salmon run on the Gisasa River in 1998 went 
480 unobserved, producing a precise total run estimate with a 95\% HPDI half width of $\pm 7.7 \%$ of the 481 estimated run size ( $\pm 29 \%$ of the total imputed missing passage). In contrast, data from the 2000

482 East Fork Andreafsky Chum salmon run indicated 17\% of the run went unobserved, producing a 483 less precise total run size estimate from the DIC-preferred $S N-N B-M A(1)$ model with a $95 \%$ 484 HPDI half width of $\pm 20.4 \%$ of the estimated run size $( \pm 118 \%$ of the total imputed missing 485 passage). The lowest precision total run estimate occurred with the 1998 East Fork Andreafsky 486 Coho Salmon run $( \pm 34.8 \%$ of the total run estimate; $\pm 112 \%$ of the total imputed missing 487 passage), which exhibited high passage count variability, strong skewness, and missing data in a 488 tail of the run (Figure $2 f)$.

The statistical arrival model total run estimates and those from linear interpolation agreed 490 closely for the case study runs that exhibited relatively smooth and symmetric arrival dynamics 491 (1998 Gisasa Chum and 1998 Gisasa Chinook; Figure 2d-e; Table 6); however, the linear 492 interpolator agreed less closely in cases with missing data near peak runs with high passage 493 variability. For example, the maximum observed count for 1998 East Fork Andreafsky Coho 494 (1,104 fish in a run where the total observed count equaled 5,417 fish) was followed by an 495 observed 0 passage day and subsequently observations ceased. Owing to a lack of observations 496 in the remainder of the run, linear imputation for the tail based upon the ad hoc rule outlined 497 above resulted in a 0 fish estimate and underestimate of the run size relative to the DIC-preferred $498 S N-N B-W N$ statistical arrival model estimate (Table 6).

500 Discussion

501 Simulation results demonstrated that in many cases missing data at weirs can be 502 accurately and precisely imputed for unimodal fish runs. For example, under the simulated 
503 process variation modeled after Pacific Salmon runs in Alaska, a weekends-off monitoring

504 schedule (a repeated schedule of 5 days of monitoring followed by 2 days of missing data)

505 produced little loss in accuracy for estimated total run size, while still allowing for estimation of

506 phenological run characteristics such as peak run date.

507 DIC-based model selection indicated support for Negative Binomial process variation for

508 all case studies, indicating that variability in daily passage counts for Pacific salmon may scale

509 positively with passage. Skewed arrival curve dynamics were supported for all but the 2000 East

510 Fork Andreafsky River Pink Salmon case study. The $S N-N B$ arrival model - process variation

511 model combination was the most flexible model estimated, requiring only one additional

512 parameter than models with Normal arrival curves. The skew-Normal arrival model reduces to

513 a Normal arrival model under a shape parameter of zero, but also has flexibility to accommodate

514 single-tail fatness through skewness. Furthermore, the Negative Binomial process model allows

515 for overdispersion in count models, but can also reduce to a pure Poisson count process model if

516 the overdispersion parameter is zero. Thus, while we recommend fitting a suite of plausible

517 statistical arrival models and subsequent DIC-based model selection, the $S N-N B$ model may be a

518 reasonable choice to apply to real data for those wishing to avoid fitting large suites of models.

519 Simulations indicated DIC-based model selection could produce ambiguous results in

520 discriminating between white noise or serially correlated process variation in some scenarios. In

521 cases of ambiguous DIC support for models with different process variation autocorrelation

522 structures, we recommend implementing models with serially correlated process variation to err

523 on the side of caution; simulation results indicate no expected loss in bias performance, and

524 while credibility intervals about imputed passage may be inflated by fitting serially correlated 
525 process variation models to data for which process variation is in reality white noise, the degree

526 of uncertainty inflation may not be great.

527 Statistical arrival model estimators outperformed linear interpolation in most simulation

528 trials; however, the performance gap was not always great, particularly when faced with data

529 with white noise process variation (Table 4). With white noise process variation simulation

530 scenarios, the proposed rule of thumb for implementing linear interpolation when the tails of a

531 fish run were missed yielded average total run estimates quite close to model-based estimates;

532 however this estimator led to a wider range in bias outcomes and occasionally led to very poor

533 estimation outcomes across simulation trials (Table 4). When faced with data simulated with

534 serially correlated process variation, the performance gap between statistical arrival models and

535 linear interpolation widened, and the proposed rule of thumb for linearly interpolating missed tail 536 areas could fail dramatically.

537 The comparable performance of linear interpolation against fitted arrival models begs the

538 question as to whether the statistical complication is worth the trouble when imputing missing

539 passages at weirs? For simple applications with only a few missing passage counts occurring at

540 non-peak passage dates, linear interpolation is likely sufficient, producing an accurate estimate of

541 total run size. The model-based framework implemented in a Bayesian framework does,

542 however, present several advantages over linear interpolation. First, particularly in data

543 scenarios exhibiting serially correlated process variation, linear interpolation can fail

544 dramatically, whereas arrival models may produce more stable imputation results. Second,

545 uncertainty estimates about missing passages are produced, providing transparency about the

546 precision of total run size. For example, the total run size estimates from the linear interpolation

547 estimator (7179) and the $S N-N B-W N$ statistical arrival model (7876) for the 1998 East Fork 
548 Andreafsky Coho Salmon were similar, differing by 697 fish; however, the statistical arrival

549 model fit indicates considerable uncertainty with a total run size 95\% HPDI ranging from 6497

550 to 11990 fish (Table 6). Third, Bayesian implementation allows for inclusion of prior

551 information into model estimates in a unified framework, which may facilitate estimates for

552 scenarios with substantial missing-data (although there is a limit to what can be asked of a

553 dataset). If multiple years' data are available for a given fish passage project, statistical arrival

554 models could be cast in a hierarchical framework, potentially improving the precision about the

555 run arrival curve parameters, and possibly improving performance of imputation by sharing

556 information across years (e.g. Hilborn and Liermann 1998; Su et al. 2001). We caution,

557 however, that in such meta-analytic approaches, care need be taken to account for potential

558 temporal or spatial trends in run timing phenology or other confounding influences on run arrival

559 dynamics when combining datasets for hierarchical inference (e.g. Thorson et al. 2013). Finally,

560 the model-based framework provides statistical estimates of biologically relevant information on

561 run arrival dynamics, where the median of the fitted arrival models represents the date at which

$56250 \%$ of the run has passed the weir, the mode represents the peak run day after which fishery

563 managers can expect declining passage counts, and the spread of the arrival model provides

564 information about fish run compression. For example, Sethi and Tanner (2014) characterized

565 annual variability in run dynamics of Chinook Salmon on the Togiak River in the Bristol Bay

566 region of Alaska, by comparing estimates of peak run date and run compression at a resistor

567 board weir across a suite of years using methods similar to the statistical arrival model approach

568 outlined above.

569 While data simulations and case studies herein focused on missed passage counts through

570 weirs, the statistical arrival model approach to imputation could also be applied to other fish 
571 enumeration techniques which produce counts that follow the underlying arrival dynamics of a

572 target fish population. Example fish counting projects include counting towers (Anderson 2000),

573 hydroacoustic enumeration (e.g. Enzenhofer et al. 1998), or test fisheries (Flynn and Hilborn

574 2004). A key requirement for such applications is that passage counts need either completely

575 enumerate all fish present on observation days, or need represent a consistent index of passage

576 on observation days (e.g. with constant or controllable "catchability").

577

\section{Acknowledgements}

579 We thank R. Brown (USFWS) and A. Martin (USFWS) for assistance with data for this project.

580 R. Brown also contributed to the conception of this project. J. Reynolds (USFWS), CJFAS

581 editorial staff and peer reviewers provided helpful comments which improved an earlier draft of

582 this piece. S. Fleischman (ADFG) is thanked for comments related to serially correlated process

583 variation. Any potential errors in the article are solely attributable to the authors. The findings

584 and conclusions in this article are those of the authors and do not necessarily represent the views

585 of the U.S. Government.

586

\section{References}

588

589 Anderson, C. J. 2000. Counting tower projects in the Bristol Bay area, 1955-1999. Alaska

590 Department of Fish and Game, Division of Commercial Fisheries Regional Information Report

591 No. 2A00-08. Anchorage, Alaska.

592 
593 Azzalini, A. 1985. A class of distributions which includes the Normal ones. Scand. J. Stat. 12:

$594 \quad 171-178$.

595

596 Bradford, M.J. 1999 Temporal and spatial trends in the abundance of Coho salmon

597 smolts from Western North America. T. Am. Fish. Soc. 128: 840-846.

598

599 Brooks, S.P., and Gelman, A. 1998. General methods for monitoring convergence of iterative

600 simulations. J. Comput. Graph. Stat. 7: 434-455.

601

602 Celeux, G., Forbes, F., Robert, C.P., and Titterington, D.M. 2006. Deviance information criteria

603 for missing data models. Bayesian Anal. 1: 651-674.

604

605 Enzenhofer, H.J., Olsen, N., and Mulligan, T.J. 1998. Fixed-location hydroacoustics as a method 606 of enumerating migrating adult Pacific salmon: comparison of split-beam acoustics vs. visual 607 counting. Aquat. Living Resour. 11: 61-74.

608

609 Fleischman, S.J., Catalano, M.J., Clark, R.A., and Bernard, D.R. 2013. An age-structured state610 space stock-recruit model for Pacific Salmon (Oncorhynchus spp.). Can. J. Fish. Aquat. Sci. 70: $611401-414$

612

613 Flynn, L., and Hilborn, R. 2004. Test fishery indices for sockeye salmon (Oncorhynchus nerka)

614 as affected by age. Can. J. Fish. Aquat. Sci. 61: 80-92.

615 composition and environmental variables 
617 Gelman, A., Carlin, J.B., Stern, H.S., and Rubin, D.B. 2004. Bayesian data analysis.

618 Chapman and Hall, Boca Raton, FL, USA.

619

620 Gewin, C.S., and VanHatten, G.K. 2005. Abundance and run timing of adult Pacific Salmon in

621 the East Fork Andreafsky River, Yukon Delta National Wildlife Refuge, Alaska, 2003. U.S.

622 Fish and Wildlife Service Data Series Report 2005-10, Anchorage, Alaska.

623

624 Greene, W.H. 2008. Econometric analysis, $6^{\text {th }}$ edition. Pearson-Prentice Hall, Upper Saddle 625 River, New Jersey, USA.

626

627 Hilborn, R., and Liermann, M. 1998. Standing on the shoulders of giants: learning from 628 experience in fisheries. Rev. Fish. Biol. Fisher. 8: 273-283.

629

630 Hilborn, R., Bue, B.G., and Sharr, S. 1999. Estimating spawning escapement from periodic 631 counts: a comparison of methods. Can. J. Fish. Aquat. Sci. 56: 888-896.

632

633 Johnson, D.H., Shrier, B.M., O’Neal, J.S., Knutzen, J.A., Augerot, X., O’Neil, T.A., and

634 Pearsons, T.A. (Eds.) 2007. Salmon field protocols handbook. American Fisheries Society, 635 Bethesda, Maryland.

636

637 Lunn, D.J., Thomas, A., Best, N., and Spiegelhalter, D. 2000. WinBUGS - a Bayesian modelling 638 framework: concepts, structure, and extensibility. Stat. Comput. 10: 325-337. 
640 Ntzoufras, I. 2009. Bayesian modelling using WinBUGS. Wiley, Hoboken, NJ, USA.

641

642 R Development Core Team (RDCT). 2014. R: a language and environment for statistical

643 computing. R Foundation for Statistical Computing, Vienna, Austria.

644

645 Sethi, S.A., and Tanner, T. 2014. Spawning distribution and abundance of a northern Chinook 646 population. Fisheries Manag. Ecol. 21: 427-438.

647

648 Spiegelhalter, D.J., Best, N.G., Carlin, B.P., and van der Linde, A. 2002. Bayesian measures

649 of model complexity and fit (with discussion). J. Roy. Stat. Soc. Ser. B 64: 583-639.

650

651 Sturtz, S., Ligges, U., and Gelman, A. 2005. R2WinBUGS: a package for running WinBUGS

652 from R. J. Stat. Softw. 12: 1-16.

653

654 Su, Z., Adkison, M.D., and Van Alen, W. 2001. A hierarchical Bayesian model for estimating

655 historical salmon escapement and escapement timing. Can. J. Fish. Aquat. Sci. 58: 1648-1662.

656

657 Thorson, J.T., Cope, J.M., Kleisner, K.M., Samhouri, J.F., Shelton, A.O., and Ward, E.J. 2013.

658 Giants' shoulders 15 years later: lessons, challenges and guidelines in fisheries meta-analysis.

659 Fish. Fish. 16: 342-361.

660 
661 Van Alen, B. W. 2000. Status and stewardship of salmon stocks in Southeast Alaska. In

662 Sustainable fisheries management: Pacific salmon. Edited by E.E. Knudsen, C.R. Steward, D.D.

663 MacDonald, J.E. Williams, and D.W. Reiser. Lewis Publishers, Boca Raton, Fla. pp. 161-193.

664 
Table 1. Prior specifications ${ }^{a}$.

Parameter description

Run size scalar

Location parameter of the Normal arrival model

Scale parameter of the Normal arrival model

Location parameter of the skew-Normal arrival model

Scale parameter of the skew-Normal arrival model

Shape parameter of the skew-Normal arrival model

Location parameter of the Student's t arrival model

Scale parameter of the Student's $t$ arrival model

Scale parameter for the Normal process error model

Dispersion parameter for the Negative Binomial process error model

Autocorrelation coefficient for lag-1 serially correlated process variation

$$
\begin{gathered}
\text { Prior } \\
\log (S) \sim \operatorname{Normal}\left(\mu_{S}=7.5, \sigma_{S}=2.0\right) \\
\mu \sim \text { Uniform }(150,300) \\
\sigma \sim \text { Uniform }(1,50) \\
\xi \sim \text { Uniform }(150,300) \\
\omega \sim \text { Uniform }(1,50) \\
\alpha \sim \text { Uniform }(-10,10) \\
\delta \sim \text { Uniform }(150,300) \\
\gamma \sim \text { Uniform }(1,50) \\
\tau \sim \text { Uniform }(0.1,1000) \\
\theta \sim \operatorname{Gamma}(0.1,0.1) \\
\rho \sim \text { Uniform }(-1,1)
\end{gathered}
$$

${ }^{a}$ Arrival models are parameterized in terms of a daily time step. For example, the prior specification for the Normal arrival model constrain the peak run date, $\mu$, to lie between Julian day 150 and day 300 . 
Table 2. Simulation and model fitting scenarios ${ }^{a}$.

$\begin{array}{ccc}\text { Observation scenario } & \begin{array}{c}\text { Arrival } \\ \text { model }\end{array} & \begin{array}{c}\text { Candidate } \\ \text { models }\end{array}\end{array}$

Simulations: white noise process variation

Weekends off: observe 5 days, miss 2 days, ...

Run peak missed: a 5-day period ( $+/-2$ days) about the run peak is missed

Late installation: first $15 \%$ of arrival curve is missed $N-N-W N$ $N-N B-W N$, $N, S N \quad S N-N-W N$, $S N-N B-W N$, LI

Simulations: $A R(1)$ and MA(1) serially correlated process variation

Late installation: first $15 \%$ of arrival curve is missed

$$
\begin{gathered}
N-N B-W N, \\
N-N B-A R(1), \\
N-N B-M A(1), \\
S N-N B-W N, \\
S N-N B-A R(1), \\
S N-N B-M A(1),
\end{gathered}
$$

Case studies: Yukon River Pacific Salmon (Oncorhynchus spp.)

$$
\begin{array}{cc}
N, S N, t(d . f . & \text { All } \\
=2) & \text { combinations } \\
& \text { (18 models })
\end{array}
$$

${ }^{a}$ Abbreviations: $\mathrm{AR}(1)=$ lag-1 autoregressive, $\mathrm{MA}(1)=$ lag-1 moving average, $\mathrm{NB}=$ Negative Binomial, $\mathrm{N}=$ Normal, $\mathrm{SN}=$ skew-Normal, $\mathrm{t}=$ Student's $\mathrm{t}$ distribution. 
Table 3. Case study data summary.

Run Observed dates

(Julian day)

1998 Gisasa River
Chum Salmon
$172-214$

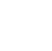
Description of missing data A priori zero passage count placement
(Julian day)
Neither tail fully observed; $\quad 158-160 ; 225-227$ sequence of missing observations preceding the run peak

\begin{tabular}{|c|c|}
\hline $\begin{array}{l}1998 \text { Gisasa River } \\
\text { Chinook Salmon }\end{array}$ & $175-214$ \\
\hline 1998 East Fork & $203-255$ \\
\hline $\begin{array}{c}\text { Andreafsky River } \\
\text { Coho Salmon }\end{array}$ & \\
\hline
\end{tabular}

Sequences of missing days

$168-170 ; 225-227$ in both tails of the run

\begin{tabular}{|c|c|c|c|}
\hline $\begin{array}{l}2000 \text { East Fork } \\
\text { Andreafsky River } \\
\text { Chum Salmon }\end{array}$ & $182-216$ & $\begin{array}{c}\text { Initial tail missed; sequence } \\
\text { of missed days after the run } \\
\text { peak }\end{array}$ & $168-170 ; 230-232$ \\
\hline $\begin{array}{c}2000 \text { East Fork } \\
\text { Andreafsky River }\end{array}$ & $182-262$ & $\begin{array}{l}\text { Sequences of missed days } \\
\text { after the run peak }\end{array}$ & $168-170 ; 270-272$ \\
\hline
\end{tabular}
Sequence of missed observations after run commencement; terminal tail missed

198-200; 270-272

Pink Salmon 
Table 4. Simulation trial performance summaries for statistical arrival models and linear interpolation under white noise Negative Binomial process variation $^{a}$.

\begin{tabular}{|c|c|c|c|c|c|c|c|c|c|}
\hline \multirow[b]{2}{*}{$\begin{array}{l}\text { Scenario } \\
\text { (arrival } \\
\text { model) }\end{array}$} & \multicolumn{7}{|c|}{ Total run size estimate percent bias } & \multirow[b]{2}{*}{$\begin{array}{l}\text { Median proportion } \\
\text { of total passage } \\
\text { observed }(\%)\end{array}$} & \multirow[b]{2}{*}{$\begin{array}{l}\text { Median } 95 \% \text { HPDI } \\
\text { width ( } \% \text { missing } \\
\text { passage prediction) }\end{array}$} \\
\hline & $\begin{array}{l}\text { Fitted } \\
\text { model }\end{array}$ & $\begin{array}{c}\text { DIC } \\
\text { preferred } \\
(\%)\end{array}$ & $\begin{array}{c}\text { Average } \\
(\%)\end{array}$ & $\begin{array}{l}\text { Minimum } \\
(\%)\end{array}$ & $\begin{array}{c}\text { Maximum } \\
(\%)\end{array}$ & $\begin{array}{l}\text { IQR } \\
(\%)\end{array}$ & RMSE & & \\
\hline \multirow{5}{*}{$\begin{array}{l}\text { Weekends off } \\
\text { (N) }\end{array}$} & $N-N$ & 0 & $<0.1$ & -11.3 & 9.6 & 5.7 & 435.5 & \multirow{5}{*}{71.6} & 28.6 \\
\hline & $N-N B$ & 70.0 & $<0.1$ & -10.7 & 10.3 & 5.8 & 426.8 & & 33.7 \\
\hline & $S N-N$ & 0 & 0.1 & -11.0 & 10.3 & 5.6 & 440.7 & & 30.3 \\
\hline & $S N-N B$ & 30.0 & 0.2 & -10.6 & 10.5 & 5.8 & 429.8 & & 33.0 \\
\hline & $L I$ & . & 0.4 & -11.4 & 12.1 & 5.8 & 501.0 & & . \\
\hline \multirow{5}{*}{$\begin{array}{l}\text { Weekends off } \\
\text { (SN) }\end{array}$} & $N-N$ & 0 & -0.8 & -15.5 & 14.5 & 8.1 & 554.6 & \multirow{5}{*}{71.2} & 26.1 \\
\hline & $N-N B$ & 1.3 & -1.0 & -13.3 & 13.5 & 7.9 & 563.6 & & 59.4 \\
\hline & $S N-N$ & 0 & -1.1 & -17.2 & 16.7 & 9.9 & 634.3 & & 26.7 \\
\hline & $S N-N B$ & 98.7 & -0.8 & -14.1 & 14.0 & 8.2 & 540.9 & & 38.8 \\
\hline & $L I$ & . & -0.4 & -13.8 & 23.2 & 8.9 & 672.4 & & . \\
\hline \multirow{5}{*}{$\begin{array}{l} \pm 2 \text { days about } \\
\text { the peak } \\
\text { missed }(\mathrm{N})\end{array}$} & $N-N$ & 0 & 0.6 & -18.7 & 20.1 & 9.6 & 668.5 & \multirow{5}{*}{74.1} & 25.9 \\
\hline & $N-N B$ & 61.3 & -0.3 & -20.3 & 17.2 & 7.2 & 604.9 & & 37.2 \\
\hline & $S N-N$ & 0 & 0.4 & -18.4 & 20.2 & 9.0 & 661.8 & & 27.5 \\
\hline & $S N-N B$ & 38.7 & $<0.1$ & -18.4 & 16.9 & 6.9 & 585.6 & & 37.3 \\
\hline & $L I$ & . & -2.5 & -24.6 & 20.5 & 11.7 & 885.3 & & . \\
\hline \multirow{5}{*}{$\begin{array}{l} \pm 2 \text { days about } \\
\text { the peak } \\
\text { missed (SN) }\end{array}$} & $N-N$ & 0 & -2.4 & -29.6 & 42.6 & 15.0 & 1144.3 & \multirow{5}{*}{63.1} & 30.6 \\
\hline & $N-N B$ & 0 & -10.5 & -37.6 & 14.1 & 10.7 & 1400.6 & & 61.6 \\
\hline & $S N-N$ & 0 & -1.2 & -34.0 & 31.4 & 14.8 & 1170.4 & & 31.3 \\
\hline & $S N-N B$ & 100.0 & -2.6 & -26.3 & 23.2 & 9.7 & 897.8 & & 46.9 \\
\hline & $L I$ & . & -6.5 & -37.7 & 25.2 & 15.6 & 1339.0 & & . \\
\hline \multirow{5}{*}{$\begin{array}{l}\text { First } 15 \% \\
\text { missed }(\mathrm{N})\end{array}$} & $N-N$ & 0 & 2.8 & -10.9 & 50.6 & 8.6 & 978.5 & \multirow{5}{*}{87.1} & 190.7 \\
\hline & $N-N B$ & 80.6 & 1.4 & -7.5 & 16.2 & 7.3 & 512.8 & & 140.8 \\
\hline & $S N-N$ & 0 & 11.4 & -12.2 & 56.0 & 24.5 & 2043.1 & & 323.8 \\
\hline & $S N-N B$ & 19.4 & 0.5 & -10.2 & 14.4 & 7.4 & 544.4 & & 181.3 \\
\hline & $L I$ & . & 0.5 & -14.8 & 176.6 & 11.7 & 2335.8 & & . \\
\hline First 15\% & $N-N$ & 0 & 6.3 & -13.4 & 74.3 & 15.9 & 1873.7 & 89.0 & 229.1 \\
\hline
\end{tabular}




\begin{tabular}{|c|c|c|c|c|c|c|c|c|}
\hline missed (SN) & $N-N B$ & 54.1 & 12.4 & -13.0 & 106.0 & 31.1 & 2662.7 & 170.4 \\
& $S N-N$ & 0 & 17.4 & -13.8 & 105.2 & 34.5 & 3016.2 & 307.3 \\
& $S N-N B$ & 45.9 & 6.3 & -13.0 & 172.6 & 12.0 & 1953.5 & 308.8 \\
& $L I$ &. & 8.8 & -14.1 & 930.0 & 10.8 & 8072.9 &. \\
\hline
\end{tabular}

${ }^{a}$ The true run size scalar for simulated fish runs is 10000 in all cases. Performance summaries for arrival model scenarios only include data sets for which all four statistical arrival model fits converged and for which a linear interpolation estimate could be generated (see Methods); number of datasets for arrival scenarios, top to bottom $=150,150,129,122,150$, and 149. Abbreviations: DIC $=$ Deviance Information Criterion, HPDI = highest posterior density interval, IQR = inner quartile range, $\mathrm{LI}=$ linear interpolation, $\mathrm{NB}=\mathrm{Negative}$ Binomial, $\mathrm{N}=$ Normal, RMSE = root mean squared error, $\mathrm{SN}=$ skew-Normal. 
Table 5. Simulation trial performance summaries for statistical arrival models and linear interpolation under white noise and lag-1 serially correlated Negative Binomial process variation ${ }^{a}$.

Total run size estimate percent bias

\begin{tabular}{|c|c|c|c|c|c|c|c|c|c|}
\hline \multirow[b]{2}{*}{$\begin{array}{c}\text { Scenario } \\
\text { (arrival model) }\end{array}$} & \multirow[b]{2}{*}{ Fitted model } & \multicolumn{6}{|c|}{ - } & \multirow[b]{2}{*}{$\begin{array}{l}\text { Median } \\
\text { proportion of } \\
\text { total passage } \\
\text { observed }(\%)\end{array}$} & \multirow[b]{2}{*}{$\begin{array}{c}\text { Median } 95 \% \\
\text { HPDI width }(\% \\
\text { missing passage } \\
\text { prediction) }\end{array}$} \\
\hline & & $\begin{array}{c}\text { DIC } \\
\text { preferred } \\
(\%)\end{array}$ & $\begin{array}{c}\text { Average } \\
(\%)\end{array}$ & $\begin{array}{c}\text { Minimum } \\
(\%)\end{array}$ & $\begin{array}{c}\text { Maximum } \\
(\%)\end{array}$ & $\begin{array}{l}\text { IQR } \\
(\%)\end{array}$ & RMSE & & \\
\hline \multirow{7}{*}{$\begin{array}{l}\text { First } 15 \% \text { missed } \\
\qquad(\mathrm{N}-\mathrm{WN})\end{array}$} & $N-N B-W N$ & 72.1 & 1.4 & -7.5 & 16.2 & 7.3 & 512.8 & \multirow{7}{*}{87.2} & 140.8 \\
\hline & $N-N B-A R(1)$ & 3.9 & 1.5 & -7.9 & 16.4 & 7.3 & 479.8 & & 154.5 \\
\hline & $N-N B-M A(1)$ & 4.7 & 1.5 & -7.8 & 16.1 & 7.1 & 485.7 & & 149.7 \\
\hline & $S N-N B-W N$ & 1.9 & 0.5 & -10.2 & 14.4 & 7.4 & 544.4 & & 181.3 \\
\hline & $S N-N B-A R(1)$ & 0 & 0.5 & -11.4 & 15.8 & 7.6 & 474.3 & & 208.2 \\
\hline & $S N-N B-M A(1)$ & 0 & 0.4 & -11.7 & 16.2 & 6.7 & 484.9 & & 198.5 \\
\hline & $L I$ & & 0.5 & -14.8 & 176.6 & 11.7 & 2335.8 & & . \\
\hline \multirow{7}{*}{$\begin{array}{l}\text { First } 15 \% \text { missed } \\
\qquad(\mathrm{N}-\mathrm{AR}(1))\end{array}$} & $N-N B-W N$ & 40.8 & 0.8 & -16.5 & 35.0 & 10.3 & 1702.3 & \multirow{7}{*}{81.8} & 139.2 \\
\hline & $N-N B-A R(1)$ & 4.8 & 0.8 & -13.3 & 32.6 & 10.0 & 1568.4 & & 207.4 \\
\hline & $N-N B-M A(1)$ & 29.2 & 0.9 & -12.8 & 31.5 & 9.6 & 1536.3 & & 198.3 \\
\hline & $S N-N B-W N$ & 24.5 & -4.7 & -24.5 & 21.1 & 9.5 & 1734.0 & & 193.5 \\
\hline & $S N-N B-A R(1)$ & 0 & -3.3 & -24.5 & 20.6 & 10.7 & 1720.9 & & 418.1 \\
\hline & $S N-N B-M A(1)$ & 0.7 & -3.9 & -24.4 & 17.8 & 11.3 & 1749.4 & & 420.1 \\
\hline & $L I^{b}$ & & 51.3 & -25.5 & 7394.7 & 12.4 & 113472.5 & & . \\
\hline \multirow{7}{*}{$\begin{array}{l}\text { First } 15 \% \text { missed } \\
\qquad(\mathrm{N}-\mathrm{MA}(1))\end{array}$} & $N-N B-W N$ & 36.7 & 4.7 & -21.6 & 71.4 & 19.7 & 1695.3 & \multirow{7}{*}{79.0} & 153.2 \\
\hline & $N-N B-A R(1)$ & 3.6 & 4.4 & -20.2 & 56.4 & 18.9 & 1345.7 & & 235.1 \\
\hline & $N-N B-M A(1)$ & 30.9 & 4.5 & -19.7 & 52.6 & 16.6 & 1311.5 & & 222.3 \\
\hline & $S N-N B-W N$ & 26.6 & -4.0 & -23.6 & 49.7 & 14.1 & 1268.5 & & 237.7 \\
\hline & $S N-N B-A R(1)$ & 0 & -1.0 & -21.6 & 39.4 & 18.8 & 1195.7 & & 537.6 \\
\hline & $S N-N B-M A(1)$ & 2.2 & -2.1 & -23.9 & 33.1 & 16.8 & 1099.2 & & 534.6 \\
\hline & $L I^{b}$ & & 125.6 & -34.6 & 16362.7 & 19.3 & 129134.6 & & . \\
\hline
\end{tabular}

${ }^{a}$ The true run size scalar for simulated fish runs is 10000 in all cases. Performance summaries for arrival model scenarios only include data sets for which all six statistical arrival model fits converged and for which a linear interpolation estimate could be generated (see Methods); number of datasets for arrival scenarios, top to bottom $=129,147$, and 139. Abbreviations: AR(1) = lag-1 autoregressive, 
$\mathrm{DIC}=$ Deviance Information Criterion, HPDI = Highest Posterior Density Interval, IQR = inner quartile range, LI = linear interpolation, $\mathrm{MA}(1)=$ lag-1 moving average, $\mathrm{NB}=$ Negative Binomial, $\mathrm{N}=$ Normal, $\mathrm{RMSE}=$ root mean squared error, $\mathrm{SN}=$ skewNormal, $\mathrm{WN}=$ white noise.

${ }^{b}$ Catastrophic failure ( $>500 \%$ bias in the total run size estimate) of the linear interpolation estimate occurred in a small number of datasets generated with serial correlated process error under the scenario of a missing tail ( $n=2$ for the $A R(1)$ scenario and $n=3$ for the MA(1) scenario). In these cases, the difference in the first observed passage count and the maximum observed passage count is small and the first observed passage count is large $(>500)$ resulting in a relatively small slope for the interpolated tail and an extremely large estimate for the run size. Removing these occasions, the average bias for the linear interpolation is $-3.5 \%$ and $-3.7 \%$ for the AR(1) and MA(1) scenarios, respectively; the maximum bias for each is $251.3 \%$ (AR(1)) and 204.9\% (MA(1)) . The RMSE for the two scenarios after removing these occasions are similarly reduced (5645.2 for the AR(1) scenario and 2654.1 for the MA(1) scenario). 
Table 6. Case study results ${ }^{a}$.

\begin{tabular}{|c|c|c|c|c|c|c|c|c|}
\hline Run & $\begin{array}{l}\text { Observed } \\
\text { passage }\end{array}$ & $\begin{array}{l}\text { Linear } \\
\text { interpolation } \\
\text { run size } \\
\text { estimate }\end{array}$ & $\begin{array}{c}\text { DIC } \\
\text { preferred } \\
\text { statistical } \\
\text { model }\end{array}$ & $\begin{array}{c}\text { Statistical } \\
\text { model } \\
\text { run size } \\
\text { estimate }\end{array}$ & $\begin{array}{c}\text { Lower } \\
95 \% \\
\text { HPDI } \\
\text { limit }\end{array}$ & $\begin{array}{l}\text { Upper } \\
95 \% \\
\text { HPDI } \\
\text { limit }\end{array}$ & $\begin{array}{l}95 \% \\
\text { HPDI } \\
\text { width }\end{array}$ & $\begin{array}{c}\text { Statistical } \\
\text { model } \\
\text { imputed } \\
\text { passage }\end{array}$ \\
\hline $\begin{array}{l}1998 \text { Gisasa } \\
\text { River Chum } \\
\text { Salmon }\end{array}$ & 13225 & 17796 & SN.NB.MA & 18010 & 16630 & 19430 & 2800 & 4785 \\
\hline $\begin{array}{l}1998 \text { Gisasa } \\
\text { River Chinook } \\
\text { Salmon }\end{array}$ & 1942 & 2361 & SN.NB.WN & 2297 & 2246 & 2357 & 111 & 355 \\
\hline $\begin{array}{c}1998 \text { East Fork } \\
\text { Andreafsky } \\
\text { River Coho } \\
\text { Salmon }\end{array}$ & 5417 & 7179 & SN.NB.WN & 7876 & 6497 & 11990 & 5493 & 2459 \\
\hline $\begin{array}{c}2000 \text { East Fork } \\
\text { Andreafsky } \\
\text { River Chum } \\
\text { Salmon }\end{array}$ & 22625 & 25089 & SN.NB.MA & 27365 & 25070 & 36260 & 11190 & 4740 \\
\hline $\begin{array}{c}2000 \text { East Fork } \\
\text { Andreafsky } \\
\text { River Pink } \\
\text { Salmon }\end{array}$ & 37069 & 43500 & t.NB.AR & 44195 & 40820 & 50970 & 10150 & 7126 \\
\hline
\end{tabular}

${ }^{a}$ Abbreviations: "DIC" = Deviance Information Criterion, "HPDI" = highest posterior density interval, "SN" = skew-Normal, "NB" = Negative Binomial, $\mathrm{t}=$ Student's t distribution (d.f.=2), $\mathrm{AR}(1)=$ lag-1 autoregressive, $\mathrm{MA}(1)=$ lag-1 moving average, $\mathrm{WN}=$ white noise. 


\section{Figure Captions}

Figure 1. Weir failures. Left panel: high water tops a resistor board weir on the Killey River, Alaska, Julne 2013 (credit: A. Waldo). Right panel: high water destroys a resistor board weir on the Gisasa River, Alaska, July 2014 (credit: J. Mears).

Figure 2. Simulated (a-c) and observed (d-h) Pacific Salmon runs. Arrival curves are constructed with posterior medians of estimated parameters from best fitting candidate models. Simulated runs include serially independent Negative Binomial process. 


\section{Figures}

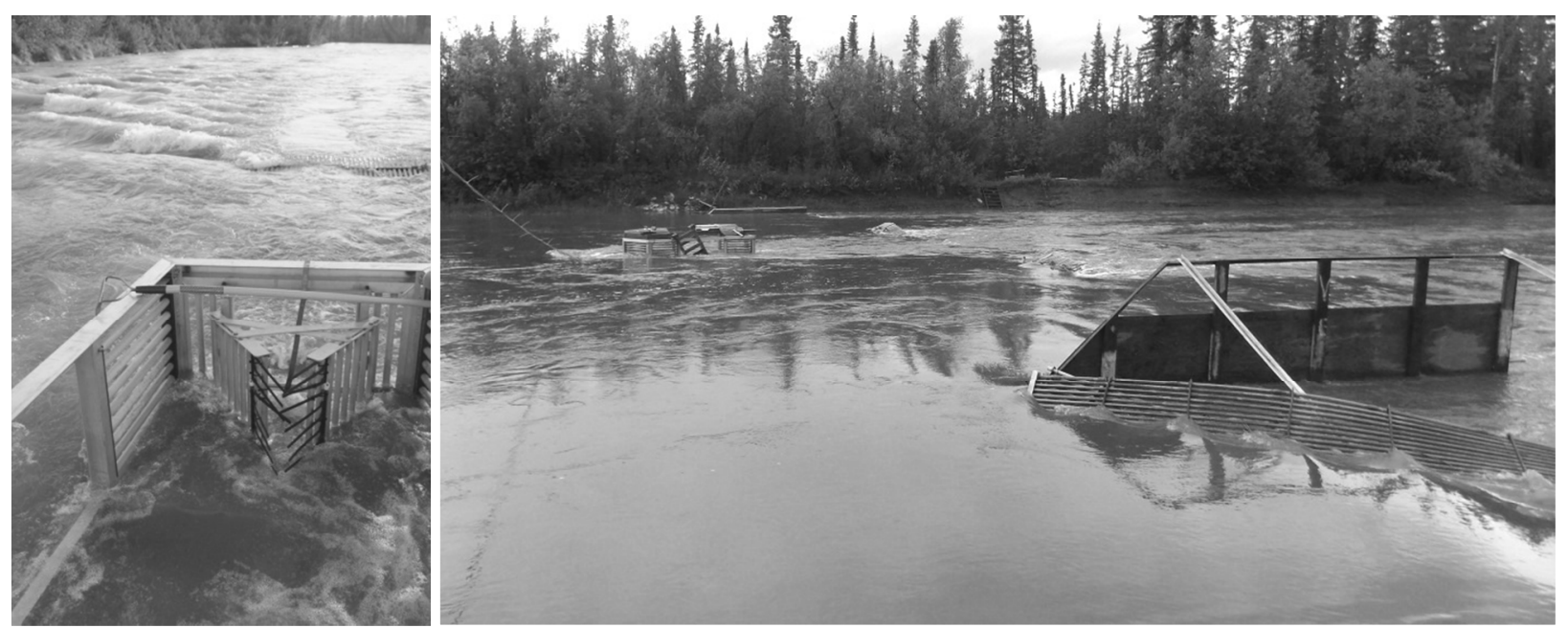

Figure 1. Weir failures. Left panel: high water tops a resistor board weir on the Killey River, Alaska, Julne 2013 (credit: A. Waldo). Right panel: high water destroys a resistor board weir on the Gisasa River, Alaska, July 2014 (credit: J. Mears). 

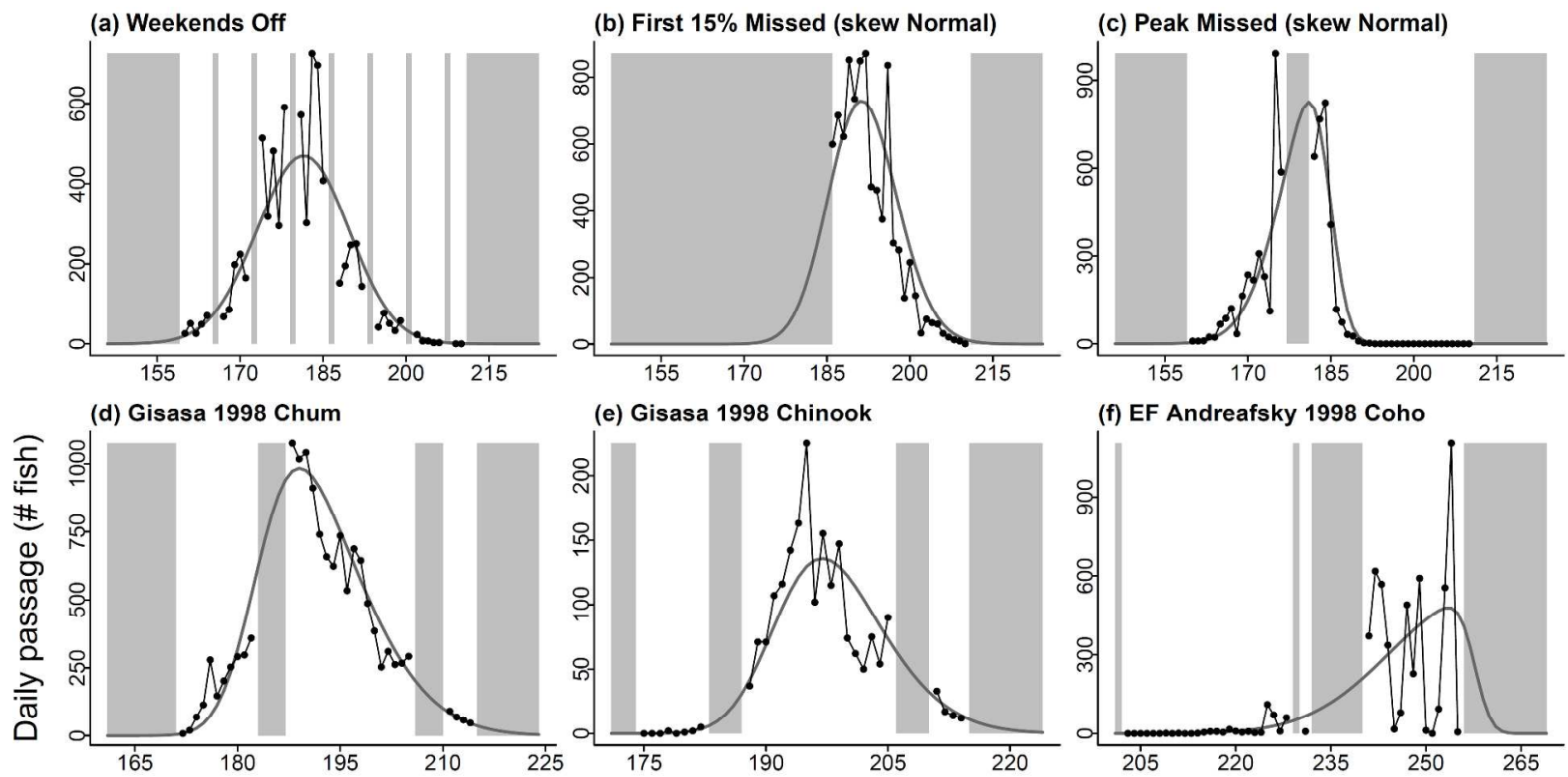

(e) Gisasa 1998 Chinook

(f) EF Andreafsky 1998 Coho
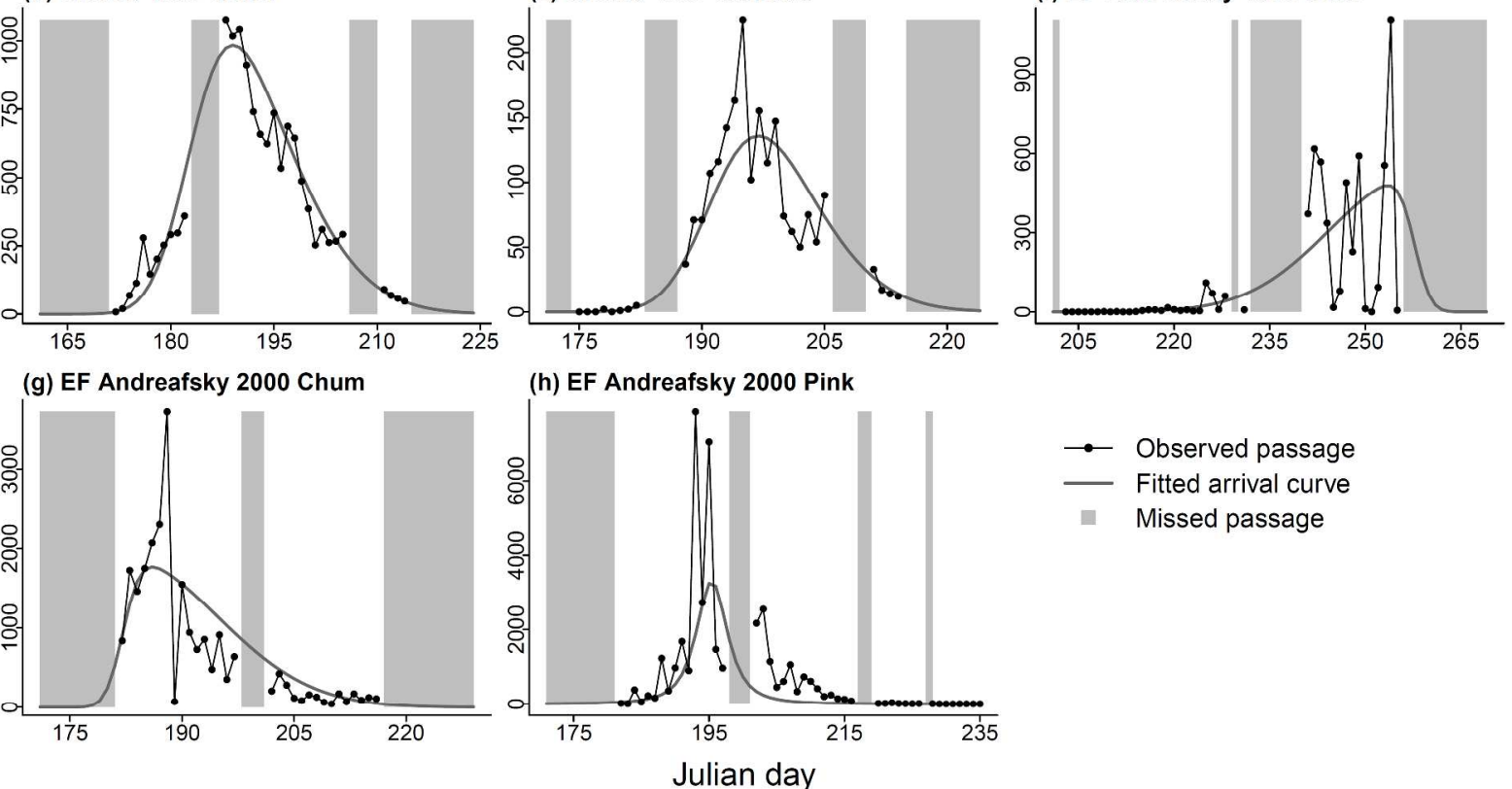
$\rightarrow$ Observed passag
- Fitted arrival curve
- Missed passage

Figure 2. Simulated (a-c) and observed (d-h) Pacific Salmon runs. Arrival curves are constructed with posterior medians of estimated parameters from best fitting candidate models. Simulated runs include serially independent Negative Binomial process. 


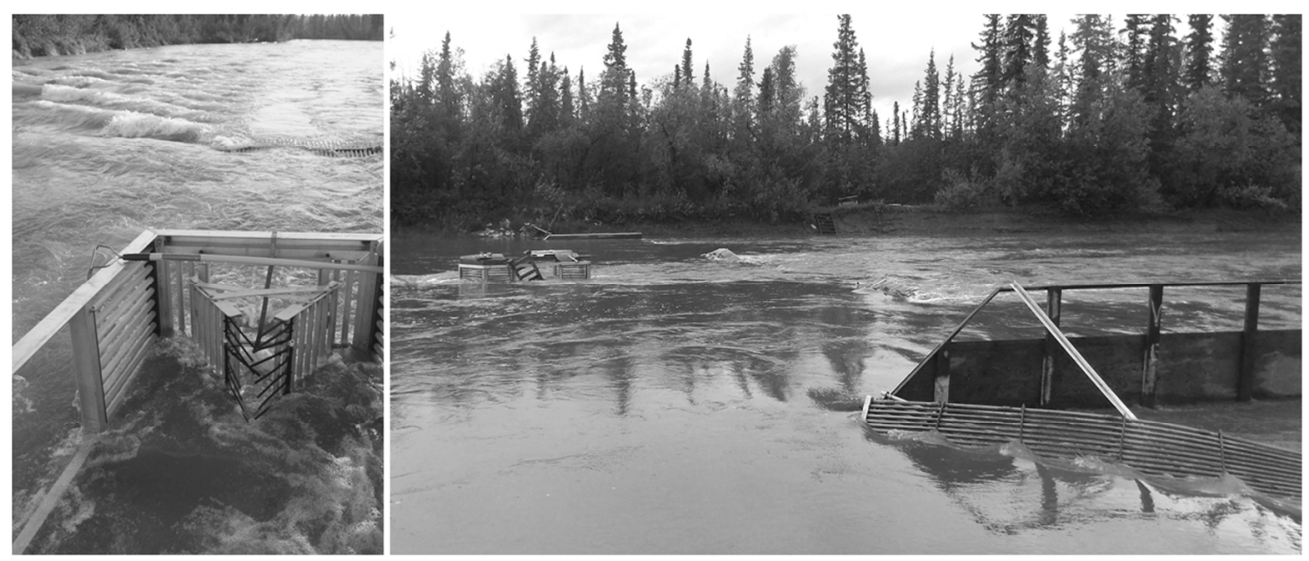


(a) Weekends Off

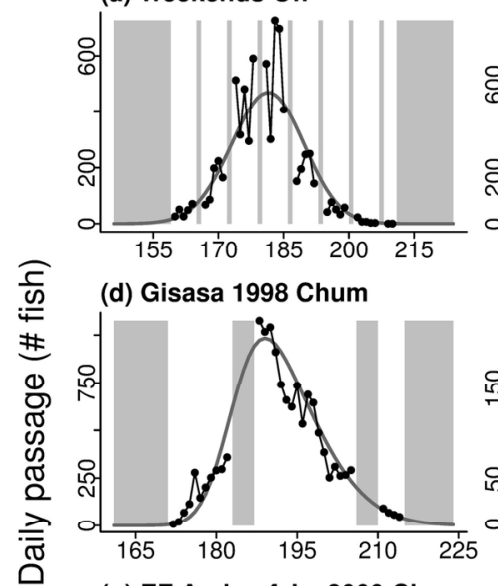

(g) EF Andreafsky 2000 Chum

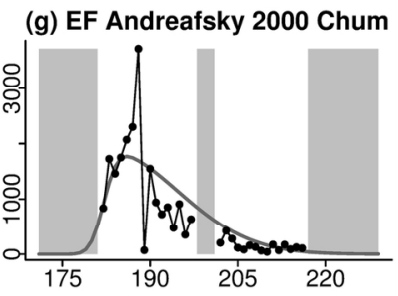

(b) First 15\% Missed (skew No

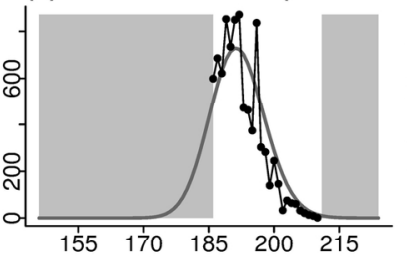

(e) Gisasa 1998 Chinook

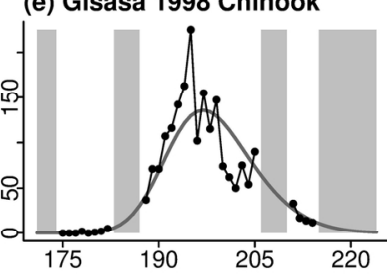

(h) EF Andreafsky 2000 Pink

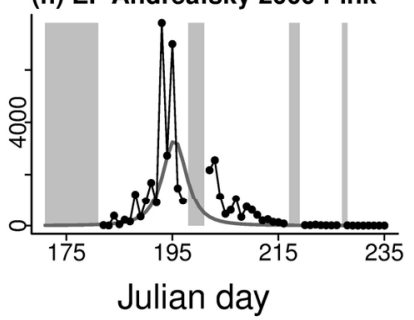

Figure 2.

$159 \times 117 \mathrm{~mm}(300 \times 300$ DPI $)$ (c) Peak Missed (skew Normal

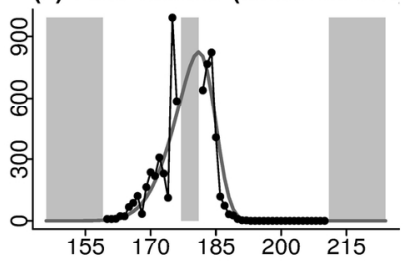

(f) EF Andreafsky 1998 Coho

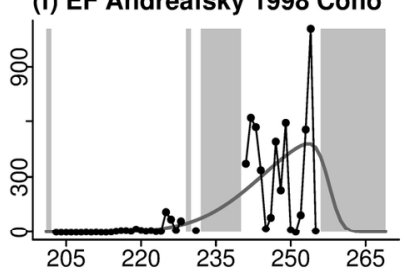

- Observed passage

Fitted arrival curve

Missed passage 